\title{
EL SISTEMA SOCIAL DE LA CASA. EN LA VIVIENDA INFORMAL CONSOLIDADA DE GUAYAQUIL
}

O sistema social da casa. No alojamento informal consolidado de Guayaquil síntese

The social system of the house. In the consolidated informal dwellings of Guayaquil

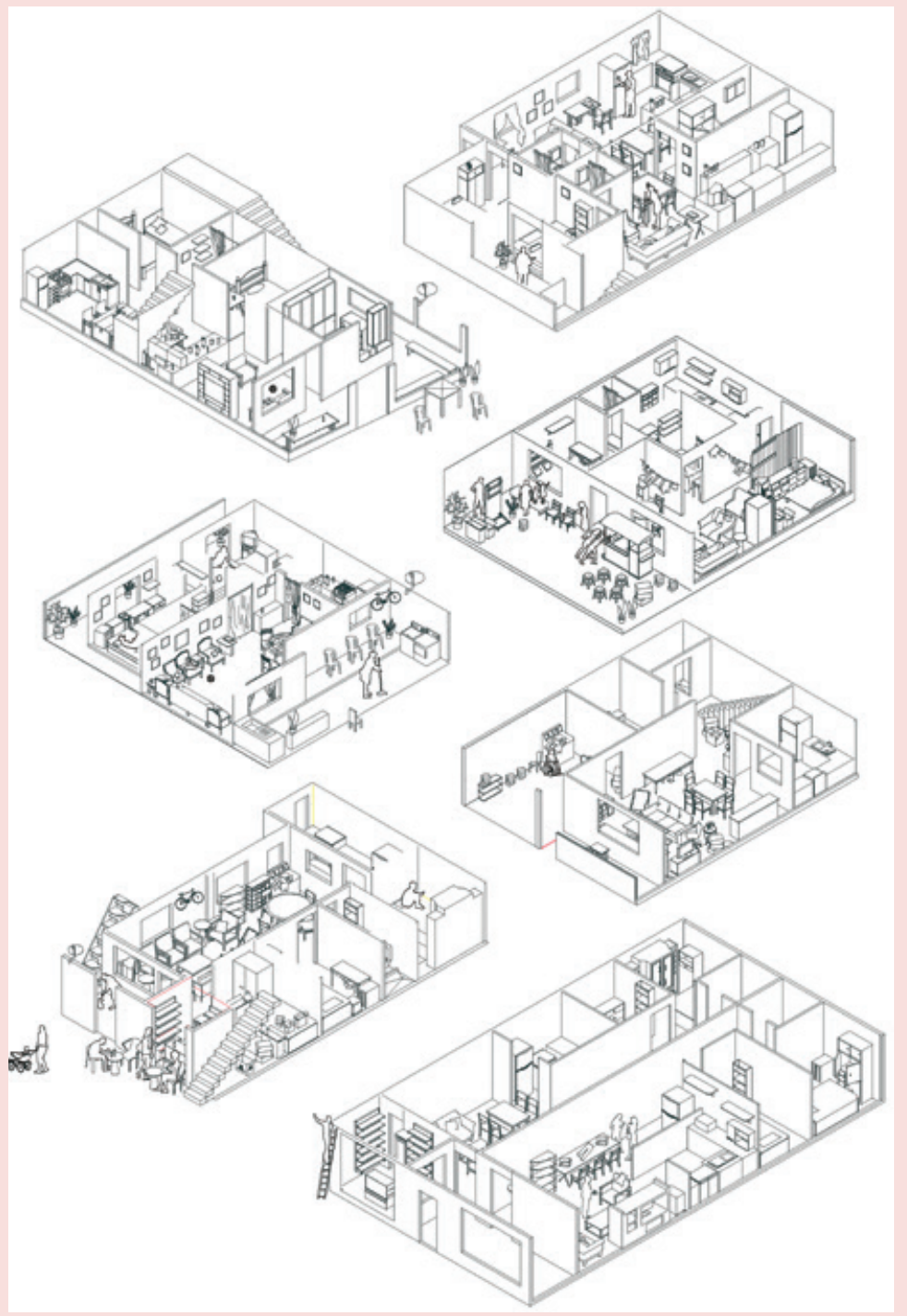

Ignacio de Teresa

Profesor ocacional Facultad de Arquitectura y Diseño.

Universidad Católica de Santiago de Guayaquil.

Guayaquil, Ecuador.

ignaciodeteresa@gmail.com

http://orcid.org/0000-0001-9136-0631

\section{Enrique Mora Alvarado}

Profesor ocacional a tiempo completo de la Facultad de Arquitectura y Diseño.

Universidad Católica de Santiago de Guayaquil. Guayaquil, Ecuador.

enriquemoraa@gmail.com

http://orcid.org/0000-0002-6003-6692

\section{Filiberto Viteri Chávez}

Docente titular auxiliar II e investigador de la Facultad de Arquitectura y Diseño.

Universidad Católica de Santiago de Guayaquil. Guayaquil, Ecuador.

filiberto.viteri@cu.ucsg.edu.ec

http://orcid.org/0000-0002-2307-4040

\section{SINDE - Universidad}

Católica de Santiago de Guayaquil. Código Presupuestario 395. Código interno 805 - 2017.

\author{
Planta baja de \\ los 7 casos de \\ estudio analizados \\ en el proyecto de \\ investigación del \\ cual este texto \\ es resultado $y$ \\ para el que se \\ para el que se \\ seleccionaron 4 . \\ del autor (2018).
}




\title{
RESUMEN
}

La crisis post pandémica de la vivienda contemporánea, desencadenada por la COVID-19, no hace sino extender a todo el planeta muchas de las cuestiones en que se hallan inmersas, de manera permanente, las viviendas en continua crisis de los países en desarrollo. ¿Cómo hacer nuestras casas más compartibles, flexibles, transformables, productivas, participativas, habitables, etc.? Por ello, un acercamiento a la vivienda de bajos recursos de estos países permite analizar alternativas a la vivienda actual, que surgen desde la informalidad como respuesta a aquellas preguntas que nos hacemos hoy todos. El siguiente documento describe parte de la investigación llevada a cabo desde la Universidad Católica de Santiago de Guayaquil, que analiza las transformaciones físicas y sociales en la vivienda informal consolidada del centro de la ciudad. Las técnicas que se utilizan incluyen el levantamiento planimétrico de casos de estudio, entrevistas a los usuarios y el mapeo del uso de las viviendas a lo largo del día. El análisis se centra en la interacción que se ejerce entre los diferentes núcleos familiares de la casa y sus objetos, llegando así a describir un sistema de transformación y producción de hábitat ligado a los objetos, en donde la vivienda es entendida como un sistema social de objetos y personas en continua interacción y transformación.

Palabras Clave: Vivienda informal, transformación social, diseño de sistemas, colectividad, viviendas multifamiliares, objetos multifuncionales

\section{RESUMO}

A crise pós-pandémica da moradia contemporânea, desencadeada pela COVID-19, apenas estende a todo o planeta muitas das questões em que estão permanentemente imersas as casas em crise contínua dos países em desenvolvimento. Surge, portanto, a seguinte questão: como tornar as nossas casas mais partilháveis, flexíveis, transformáveis, produtivas, participativas, habitáveis, etc.? Neste sentido, uma abordagem dos problemas habitacionais de populações de baixa renda nestes países permite analisar alternativas aos modelos de moradia atuais que emergem da informalidade como uma resposta às questões que todos nos colocamos hoje. O seguinte documento descreve parte da pesquisa realizada pela Universidade Católica de Santiago de Guayaquil que analisa as transformações físicas e sociais na estrutura habitacional informal consolidada do centro desta cidade equatoriana. As técnicas utilizadas incluem o levantamento planimétrico de casos de estudo, entrevistas aos usuários e o mapeamento do uso das estruturas ao longo do dia. A análise se centra na interação entre os diferentes núcleos familiares da casa e seus objetos, descrevendo, assim, um sistema de transformação e produção de habitat ligado aos objetos no qual a estrutura habitacional é entendida como um sistema social de objetos e pessoas em contínua interação e transformação.

Palavras-Chave: Moradia informal, transformação social, concepção de sistemas, coletividade, habitação multifamiliar, objetos multifuncionais

\begin{abstract}
The post-pandemic crisis of contemporary housing, triggered by COVID-19, only but extends, to the entire world, many of the questions in which, permanently, housing is immersed within, in an ongoing crisis of developing countries. How to make houses more shareable, flexible, transformable, productive, participatory, livable, etc.? In that sense, by studying low-income housing in these countries, it is possible to analyze alternatives to the current dwellings, that arise from informality as a response to those questions shared worldwide today. This article describes part of a research carried out at Universidad Católica de Santiago de Guayaquil, which analyzes the physical and social transformations in consolidated informal dwellings within the city center. The techniques used, include planimetric surveys of case studies, interviews to users, and mapping out the use of the dwellings throughout the day. The analysis focuses on the interaction exerted between several nuclear families inside the dwelling and their objects. Thus describing a habitat transformational and production system linked to objects, where the dwelling is understood as a social system of objects and people, in continuous interaction and transformation.
\end{abstract}

Keywords: Informal housing, social transformation, systems design, collectivity, multifamily housing, multifunctional objects 


\section{INTRODUCCIÓN}

1 Desde la Universidad Católica de Santiago de Guayaquil UCSG varios proyectos de investigación han abordado la relación entre el crecimiento progresivo de las familias y la transformación de sus viviendas y del espacio público colindante (Mora, 2013, de Teresa, 2015, Mora, Viteri y De Teresa, 2017), dando como resultado varios artículos y una tesis doctoral en la Universidad de Granada, "Sistemas de Transformación en la Vivienda Informal Consolidada: caso de Santa María de las Lomas, Guayaquil" (De Teresa, 2017). El presente artículo se apoya fundamentalmente en los resultados del proyecto "Estudio de la generación de colectividad en la vivienda informal consolidada: caso Santa María de las Lomas, Guayaquil" (Mora et al., 2017).

2 El barrio de Santa María de las Lomas está situado en el centro geográfico de Guayaquil, encerrado entre la avenida Barcelona, la ciudadela La Fuente, y la UCSG. Está formado por unas 200 viviendas, que prácticamente en su totalidad albergan más de un núcleo familiar. El primer asentamiento tuvo lugar a finales de la década de los 50, producto del desalojo y reubicación de un grupo de pobladores de la zona de La Atarazana, a los que se les construyeron unas primeras 50 casetas de caña. Las familias se organizaron inicialmente a través de un Comité Barrial y más tarde, en 1969, en una Cooperativa. Sin embargo, la legalización no tuvo lugar hasta 1980, a pesar de que el abastecimiento formal de servicios públicos — pavimentación de calles, agua potable, alcantarillado y electricidad- Ilegó después (De Teresa, 2015).

3 En la construcción informal, los muros de ladrillo suelen utilizarse como apoyo para las vigas de hormigón, por lo que se pierde la independencia estructural respecto a los cerramientos (Hernández, Kellet y Allen, 2012, p. 29).
El presente artículo expone parte de los resultados de una investigación de la Universidad Católica de Santiago de Guayaquil que analiza la interacción entre los usuarios de la vivienda y sus objetos, así como la influencia que tienen ambos conjuntos en la generación de encuentros dentro y fuera de la casa ${ }^{1}$. A través de preguntas como ¿qué relación hay entre la casa, sus cosas y las personas que la ocupan?, o ¿qué transformaciones sufren las diferentes agrupaciones de personas y objetos?, la investigación analiza varios casos de un barrio de vivienda informal consolidada de Guayaquil.

El barrio de estudio, Santa María de las Lomas, es uno de los asentamientos informales que han quedado inmersos en la trama urbana de la ciudad. Producto de una reubicación, su consolidación se ha desarrollado durante seis décadas al margen de la normativa municipal, siendo autorregulada a través de comités barriales y cooperativas vecinales ${ }^{2}$. Este proceso ha estrechado los lazos entre las distintas familias. A su vez, el terreno de asentamiento es contiguo al campus de la Universidad Católica de Santiago de Guayaquil. Con ello, surgió y se fortaleció con los años una relación simbiótica entre ambos ámbitos, dando lugar a interesantes relaciones supra-familiares.

Ejemplo de lo anterior lo constituyen los estudiantes y trabajadores de la universidad, que comen diariamente en alguna de las viviendas del barrio, o practican deporte en la calle junto a los vecinos. Las familias se organizan para trasladar parte del mobiliario fuera de sus casas y llenar la calle temporalmente con objetos como mesas, carpas, cocinas rodantes, piscinas, porterías de fútbol, redes de volley, etc. Se establece así una relación directa entre estas agrupaciones de objetos provenientes de varias viviendas y los encuentros entre personas del barrio y de la universidad.

Esta capacidad de los objetos para agruparse al ritmo de las personas contrasta radicalmente con la rigidez de la casa, la cual sufre las limitaciones de un sistema constructivo importado e ineficaz. Un siglo después de su aparición, el modelo de vivienda planteado por la modernidad sigue prevaleciendo. El sistema Dominó ha sido aceptado a escala planetaria como paradigma de la arquitectura de la casa, a pesar de la rigidez de su estructura de hormigón que, en su versión bricolaire, pierde su independencia estructural, renunciando definitivamente a la prometida flexibilidad de contenido ${ }^{3}$. La modernidad irrumpió en el territorio doméstico como una "obra de purificación" que se oponía a la existencia de objetos impuros e híbridos. En consecuencia, tanto su sistema constructivo y espacial, como normativo (a escala de casa y de ciudad), se resisten a la libre transformación, relegando las posibilidades de cambio al interior de la habitación.

Esto hace que la transformación de la vivienda quede habitualmente restringida por la normativa y que, en caso de permitirse cambios, éstos sean muy difíciles de ejecutar en la práctica. La transformación se hace aún más complicada en la vivienda informal, en donde la autoconstrucción —acelerada por la urgencia de la ciudad_ da 
4 En su libro Factfulness, Hans Rosling argumenta que, en estos casos donde no se suele tener acceso a un banco, la mejor manera que tienen los propietarios de invertir con los ingresos discontinuos que van obteniendo es comprando ladrillos que incorporan a su vivienda. De esta manera, además de ir mejorando su casa poco a poco, se aseguran de que nadie les robara sus ladrillos, y que éstos no se devaluarán con el tiempo, como ocurre con el dinero (2019, p. 188).

5 Los parámetros que determinan el concepto de "consolidación" de la vivienda informal vienen desarrollados en el artículo "Aproximaciones familia-casa" (De Teresa, 2016a).

6 Rafael Iglesia (2011) describe cómo la lenta transformación a lo largo de los siglos de ciertos objetos cotidianos, como martillos - llaves inglesas, hasta llegar a ser lo que son hoy, está basada en una crítica continua a un modelo precedente, siguiendo un proceso más natural que artificial. como resultado torpes reestructuraciones para intentar añadir una nueva habitación, una nueva planta, dividir el acceso en dos, etc.

Sin embargo, la voluntad de transformación no es exclusiva de la vivienda informal. Las restricciones sanitarias que ha traído consigo el COVID- 19 han generalizado aquellas cuestiones a las que ya se enfrentaban las viviendas en permanente crisis de los países en desarrollo. Problemáticas que afectan a las viviendas de bajos recursos, como la rigidez del modelo de hábitat o su incapacidad para reaccionar de manera inmediata a los nuevos cambios, han llegado hoy a ser universales.

El modelo moderno, y su pretendida pureza formal, se ha ido deliberadamente distanciando de una creciente cultura híbrida y desigual, en donde el cruce con otras disciplinas no arquitectónicas se plantea imprescindible para el desarrollo e innovación en materia de vivienda. La vivienda demanda capacidad para incorporar con facilidad los últimos avances, cualesquiera que sean: programáticos, sociales, productivos, ambientales, tecnológicos, energéticos, de gestión de residuos, etc. La innovación e invención deben poder llegar de manera inmediata al espacio doméstico y, especialmente tras la pandemia, los usuarios en todo el mundo demandan ser partícipes directos de estas incorporaciones.

La vivienda informal, debido a su continuo estado de crisis, ha reclamado desde siempre esta necesidad de transformación inmediata. Por lo general, sus usuarios no tienen acceso a créditos bancarios con los que financiar la construcción de su casa de una sola vez, y necesitan ir añadiendo cambios poco a poco ${ }^{4}$. Frente a la rigidez de la casa para incorporar estos cambios, los muebles y demás objetos parecen tomar la responsabilidad de transformar el uso del espacio. En lugar de añadir una nueva habitación, los objetos se encargan de subdividir el espacio interior o de hibridar los usos.

La casa, por un lado, y sus cosas, por otro, tienen por ello naturalezas diferentes, habiendo llegado incluso a ser opuestas. Si bien la casa pretende ser un único objeto perfecto e indeformable, los numerosos objetos de su interior parecen formar un sistema de elementos que, probablemente como reacción, se desplazan y reagrupan incesantemente. Por este motivo, los núcleos familiares y objetos de la casa son el centro de esta investigación que pretende averiguar el papel que pueden tener éstos últimos en la transformación del sistema de vivienda.

La vivienda informal consolidada ${ }^{5}$, y su manera de sumar cambios al margen de la normativa, se torna crítica directa del modelo de vivienda moderna; crítica lenta, que durante décadas ha ido alterando, de manera ininterrumpida, la forma y uso de la casa ${ }^{6}$. Una que ha ido evidenciando y dando respuesta a problemáticas como la convivencia de varios núcleos familiares en una misma casa o la necesidad de generar ingresos. Representa, por ello, un valioso campo de experimentación, en donde poder analizar la transformación que ha sufrido la familia, junto con la casa, como partes de un sistema social en constante evolución. 


\section{METODOLOGÍA}

7 En este texto se define a la "vivienda" como el sistema de hábitat compuesto por diferentes objetos entre los que la "casa" es el mayor de todos. La "casa" es entendida por ello como un objeto más, cuya relación con el resto de objetos es por inclusión. La "vivienda" engloba a la casa y al resto de mobiliario y objetos domésticos.

8 Santiago de Molina analiza bajo el título "La invasión de los objetos" (2013), el papel de éstos en el desarrollo de la vida doméstica. En su tesis sobre la "Arquitectura Collage" (2014), desarrolla a su vez este concepto de arquitectura como suma de acontecimientos.
El presente trabajo muestra cuatro de las siete viviendas analizadas en el proyecto de investigación. Estos sintetizan los principales hallazgos y permiten extraer conclusiones a través de la comparativa entre ellos. Tanto las transformaciones sociales que ha sufrido cada uno de los cuatro grupos familiares analizados, como las modificaciones físicas de sus viviendas, son únicas. Esto hace que también se modifique la manera en que se franquean los límites físicos y sociales impuestos por la casa ${ }^{7}$ y por la familia.

Los cuatro grupos familiares analizados están formados por más de un núcleo; su número de personas y estructura social también es diferente, así como el proceso de transformación que han sufrido desde su origen. Lo mismo ocurre con sus viviendas, que difieren en tamaño, organización espacial y relación con el entorno cercano. Cada caso expuesto presenta por eso características particulares que afectan a su organización física y social: unos crean densas redes intrafamiliares y otros relaciones supra-familiares con los vecinos del barrio y con el personal de la vecina universidad; unos casos trasladan objetos al exterior de la casa y otros introducen objetos públicos al interior de la vivienda.

En la Figura I se muestra la ubicación de los cuatro casos de estudio seleccionados en el barrio:

- Caso Nol: tres núcleos familiares / siete integrantes / vivienda de una sola planta que no ha sufrido grandes modificaciones a lo largo del tiempo.

- Caso N²: cinco núcleos familiares / nueve integrantes / vivienda de tres niveles con grandes transformaciones desde su origen.

- Caso N³: dos núcleos familiares / nueve integrantes / vivienda de tres pisos con cambios significativos.

- Caso N4: (Inquilina) / dos núcleos familiares / tres integrantes / vivienda con locales comerciales en planta baja pertenecientes a la familia.

La investigación analizó los encuentros entre diferentes núcleos familiares, las viviendas, sus espacios y objetos, tanto dentro como fuera de la casa. Para ello, se llevaron a cabo levantamientos planimétricos detallados (plantas, secciones y axonometrías) de las viviendas y del mobiliario doméstico ${ }^{8}$, y se condujeron entrevistas semiestructuradas a los usuarios para analizar su actividad a lo largo del día. En paralelo, se observaron los desplazamientos y agrupaciones de personas y objetos, con el objetivo de develar la influencia que tenían estos últimos en la generación de actividades colectivas. Finalmente, se condujeron entrevistas con el propósito de revelar el desplazamiento y actividades que realiza cada miembro de la familia, el espacio en el que estas actividades suceden y los objetos con los que interactúan. En conjunto, planos, entrevistas y observaciones, permitieron elaborar mapas de desplazamientos e interacciones entre las personas y los objetos durante la mañana, tarde y noche [Figura 2]. 


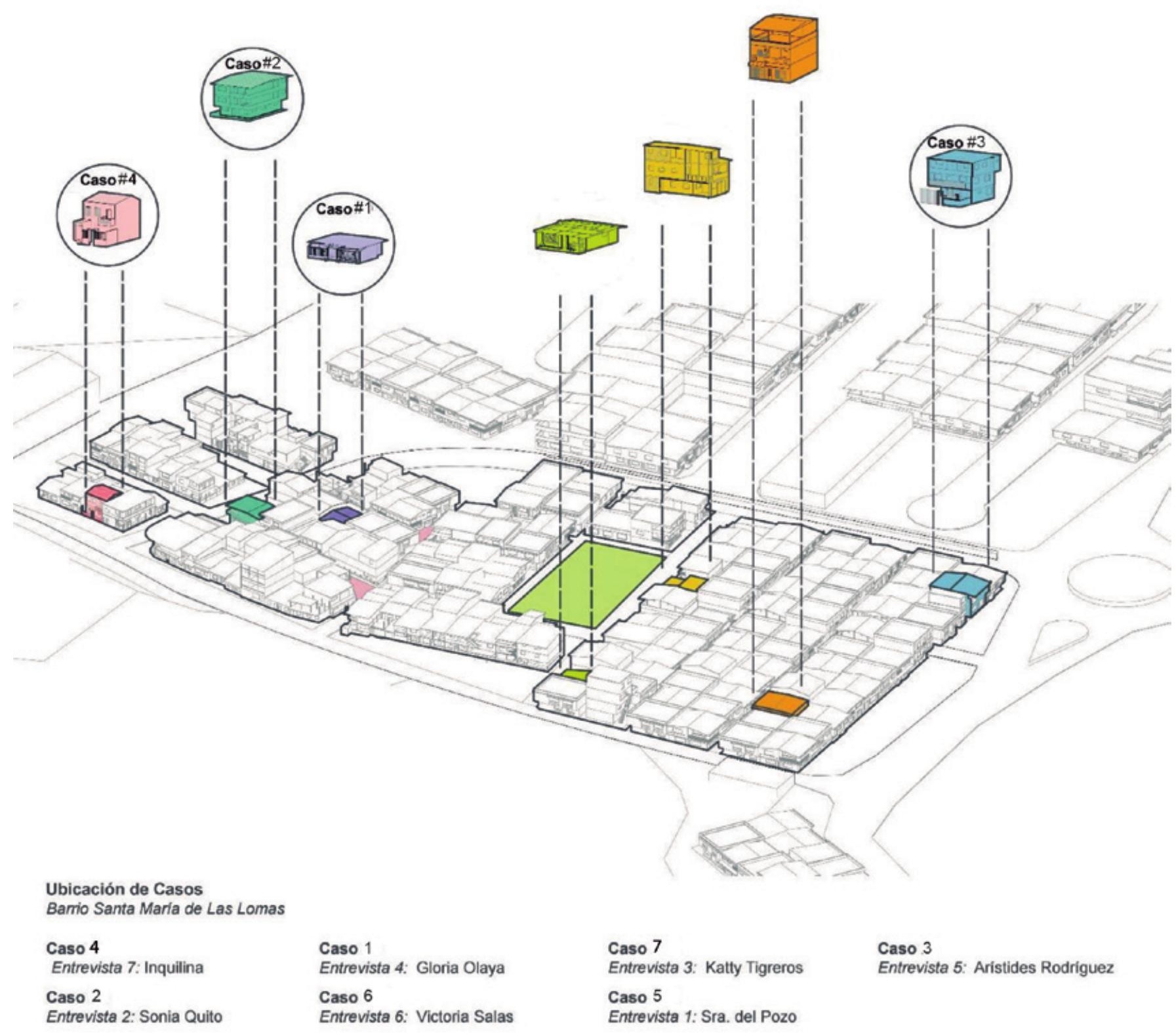

Figura 1

Ubicación de los casos seleccionados en el barrio Santa María de las Lomas Fuente: Fotografía Fuente: Fotografía
del autor $(2018)$.
Tanto en los mapas de uso, como en el resto de la planimetría, se han considerado a las personas y a los objetos como sujetos de representación gráfica por igual. De esta forma, las personas y sus objetos han sido entendidos como conjuntos con influencia mutua. Se ha llegado a evidenciar el rol que tiene el sistema de objetos de la casa en la generación de actividades y cómo su desplazamiento es capaz de modificar el uso del espacio en cada caso. Los planos muestran tanto el interior de las viviendas, como su entorno inmediato hacia la calle; así, se pueden analizar las actividades que ocurren en el borde entre ambos, y los encuentros que aparecen entre los usuarios de la vivienda y sus vecinos cercanos, o con personas de fuera del barrio. 
Figura 2

Axonometrías

comparativas de

los espacios y

mobiliarios de mayor

uso en el interior

de la casa

Fuente: fotografia

del autor (2018).

\section{\# 1}

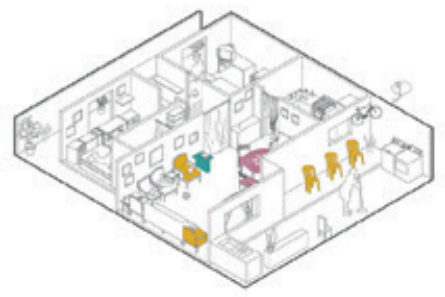

NÚCLEO FAMLLIAR
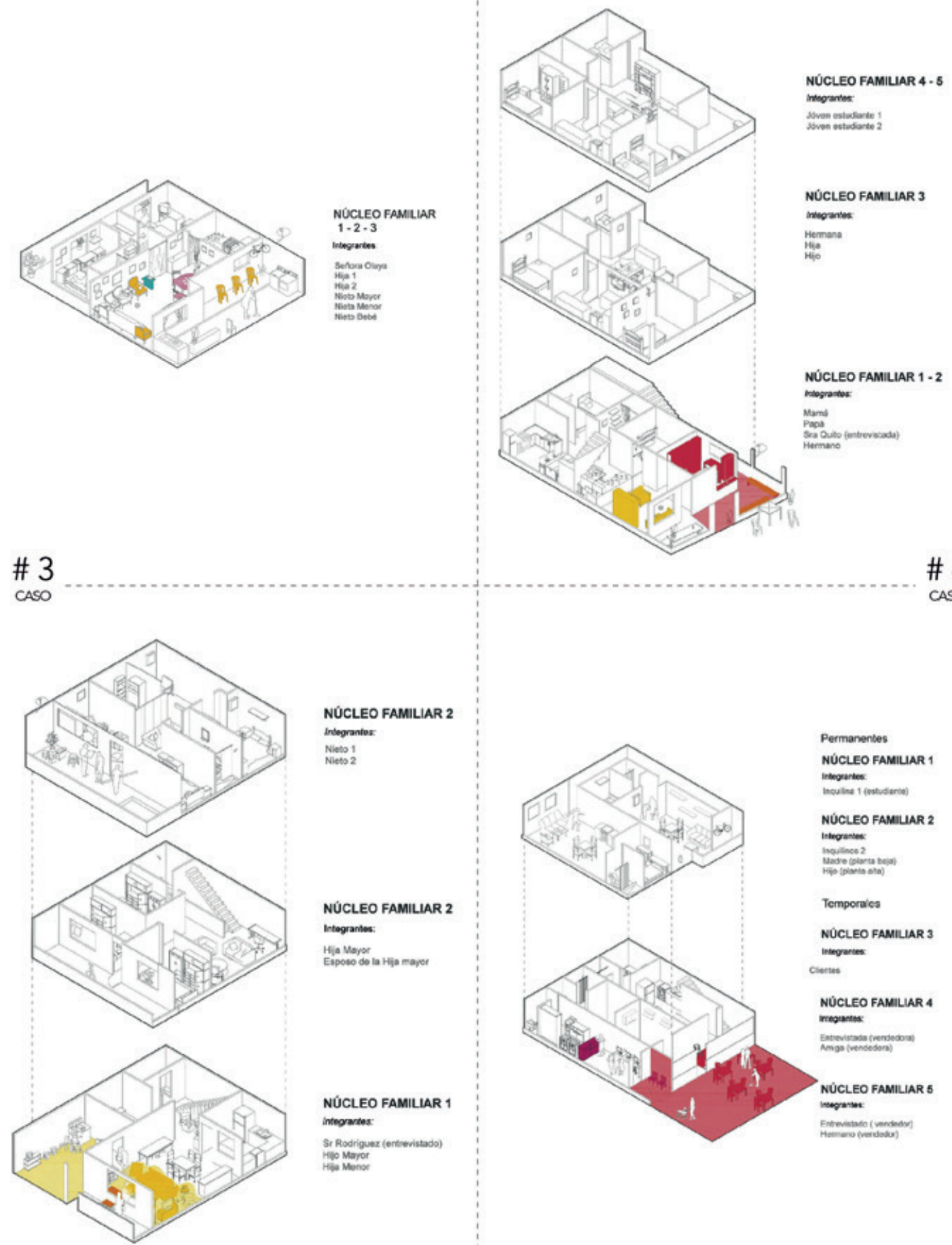

NÚCLEO FAMILIAR 2

\section{Ninegran 1 .}

NÚCLEO FAMILIAR 2

Intagranter.

Hipono do la Hilla mayer

NÚCLEO FAMILIAR 1

intogravitos:

Git Rodriguez (enteceistado)

Hilo Maror 
Figuras 3 y 4

Excursiones.

Talleres de pintura con los estudiantes cocinas rodantes para las meriendas de la noche, carpas, merenderos improvisados con jabas de plástico, jabas de plástico, ecuavoley y el ecuavoley y el cartel de reglas farola que ilumina la cancha, etc.

(parecido al coche) Fuente: Fotografía del autor (2019).
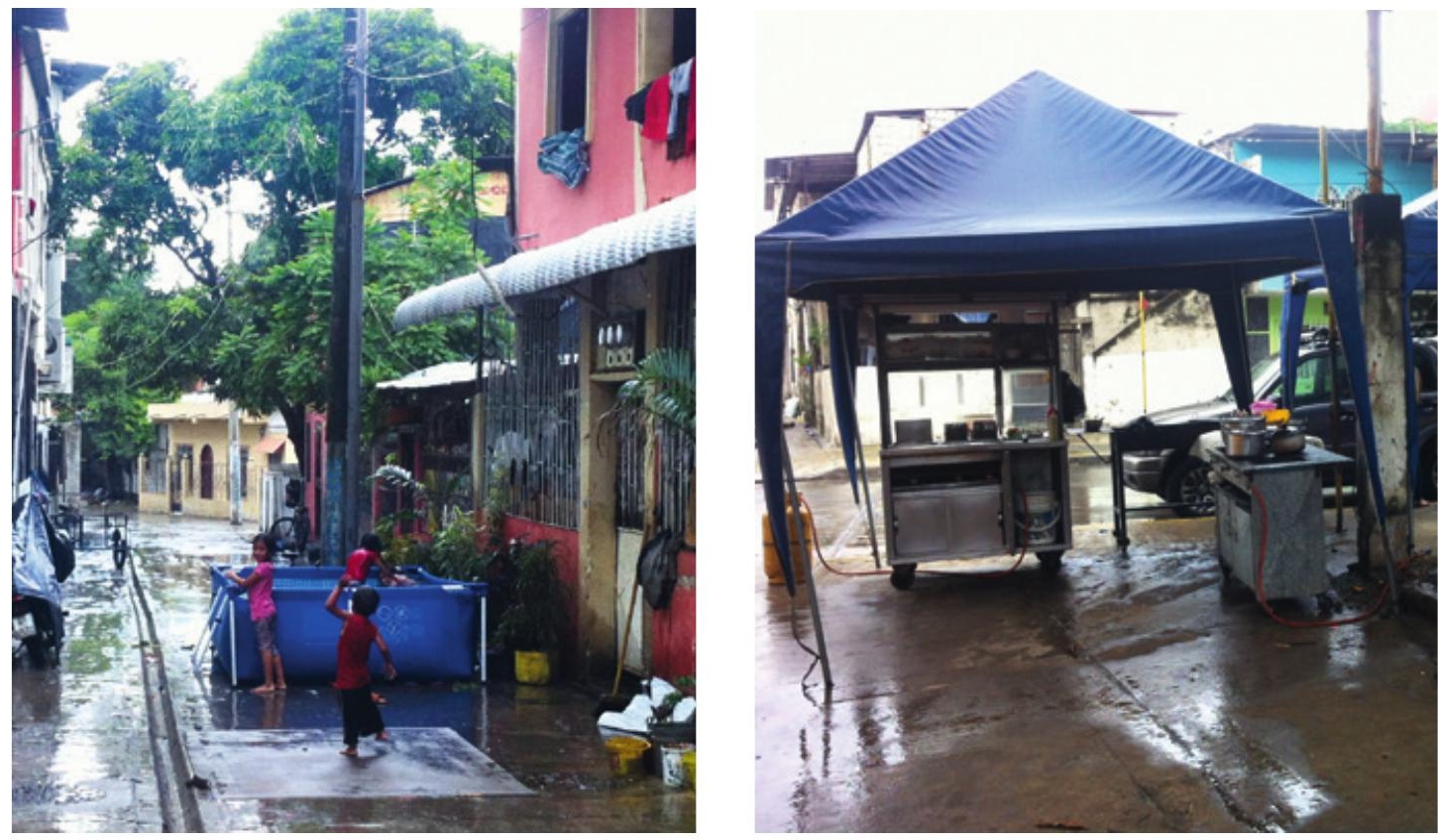

INCURSIONES

AS / Vol. 39. No59 / ENERO 2021 // ISSN impresa 0716-2677 / ISSN digital 0719-6466 


\section{EXCURSIONES}

AS / Vol. 39. N059 / ENERO 2021 // ISSN impresa 07 I 6-2677 / ISSN digital 0719-6466 con una tienda/bar. Además, en ciertos momentos, los usuarios de la tienda ingresan a la vivienda cuando hacen uso del baño, extendiendo la actividad hacia el interior de la casa y compartiendo con la familia en los espacios privados. El caso $\mathrm{N}^{0} 3$ es el que tiene menor grado de incursión, puesto que se trata de una tienda que se vincula con el exterior a través de una ventana. Finalmente, en el caso $N^{\circ} 4$, se aprecia que la planta baja, al estar destinada a una actividad netamente comercial (cyber-café), es la que permite en mayor medida el flujo de personas entre el interior y exterior de la vivienda.

Estas incursiones se producen por actividades sociales — celebración de misas, rifas, o bingos-, normalmente con vecinos del barrio, y actividades comerciales — en bares o tiendas adaptadas - que incluyen adicionalmente a la comunidad universitaria, y ocurren gracias a la flexibilidad interior de la vivienda, que es capaz de alterar su programa inicial acogiendo nuevos usos a lo largo del día. Las viviendas recurren, a menudo, a elementos móviles como cortinas o paneles para resolver situaciones imprevistas, separando visualmente zonas de la casa o, en su defecto, al traslado temporal de objetos entre las diferentes instancias. Es decir, la fragmentación espacial que había propuesto el programa en un principio es posteriormente alterada por el uso real. Las axonometrías de las viviendas [Figura 2] muestran este protagonismo del objeto frente al contenedor.

Las Figuras 3 y 4 ilustran el traslado temporal de objetos sobre la calle: carpas, cocinas, piscinas, mesas o sillas. Dichos objetos son los responsables de la generación de actividades comunitarias al exterior de la vivienda. Lo que comienza como un espacio público apropiado temporalmente por una única familia, se llena de otras y adiciona estudiantes universitarios. Las familias no poseen el espacio interior suficiente para brindar servicios, ni tienen acceso al financiamiento para incorporarlos en adiciones dentro de su lote particular. Por consiguiente, optan por acomodar en el exterior los objetos necesarios para la actividad comercial de su interés.

De los casos seleccionados [Figura 5], el $\mathrm{N}^{\circ}$ I es el que presenta menor grado de relación de la vivienda con el exterior. Sin embargo, por la tardes y noches la familia utiliza la acera para la venta de comidas rápidas, incorporando una pequeña cubierta desmontable. En el caso $\mathrm{N}^{\circ} 2$, se observa que la tienda que funciona al interior de la vivienda dispone de un pequeño espacio con sillas en la acera. No obstante, alrededor de la casa se dan aglomeraciones de personas los fines de semana y por las noches. Esto se debe a que la familia, en conjunto con sus vecinos, aprovechan las aceras y calles para organizar bingos. En el caso $\mathrm{N}^{\circ} 3$, la familia hace uso de una zona de parqueo público e incorpora unas bancas municipales para vender comida. Finalmente, en el caso $\mathrm{N}^{0} 4$ - el más próximo a la entrada de la universidad-, se observan carretas de comida, sillas y mesas que la familia y vecinos disponen en la calle, que generan un corredor comercial para brindar servicios a los estudiantes universitarios. 


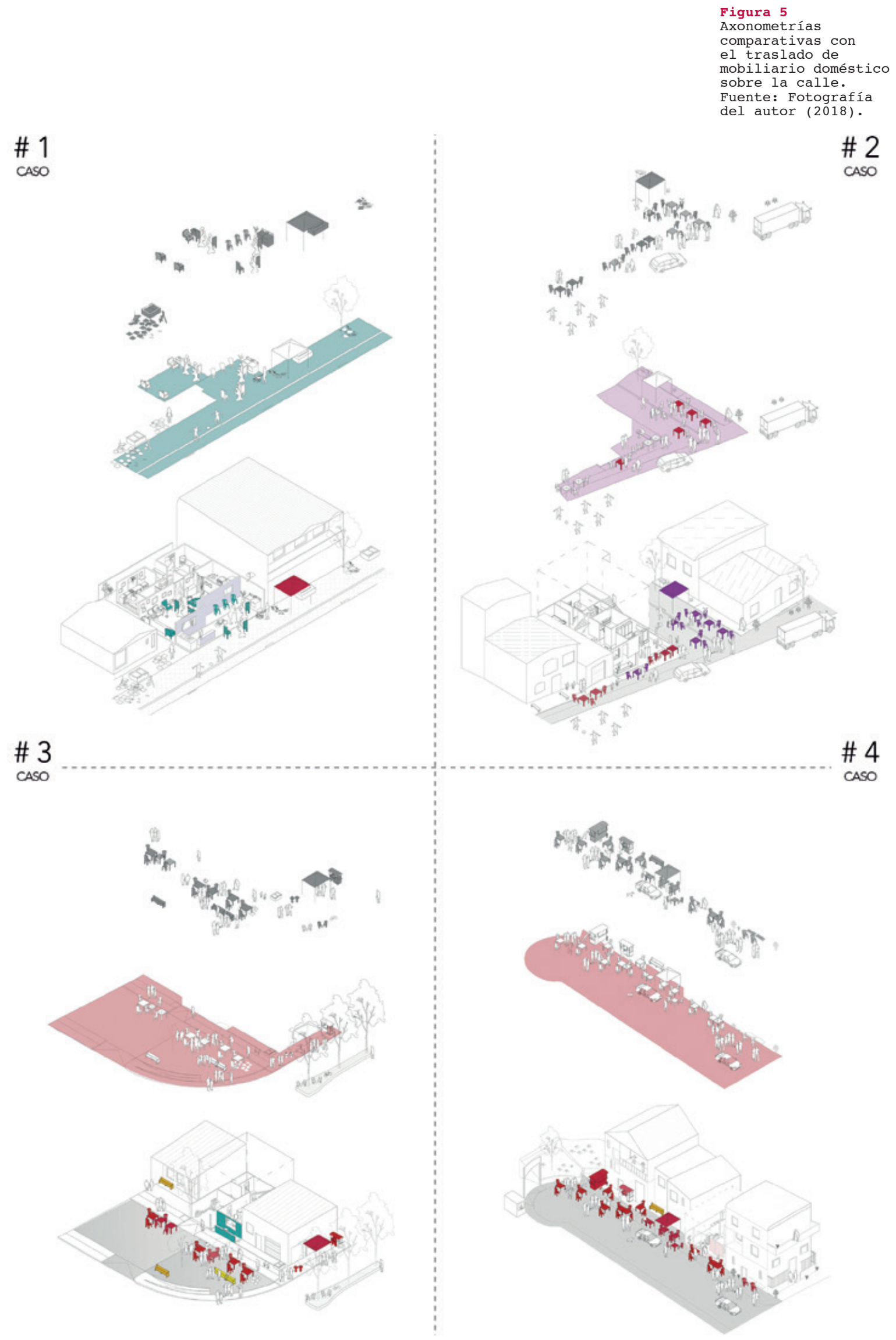




\section{BORDES}

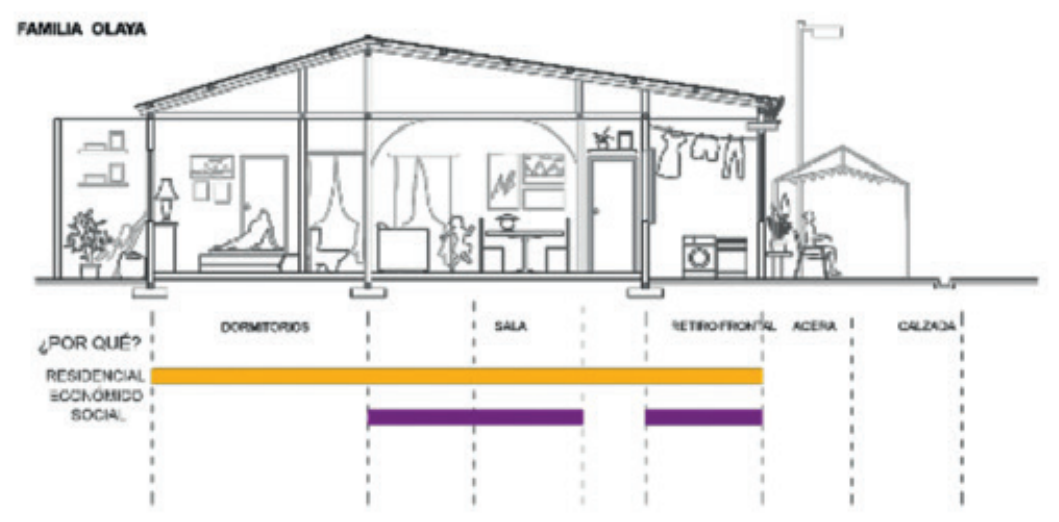

Al reagrupar objetos y personas de diferentes viviendas, las incursiones y excursiones transgreden y desdibujan el límite que establece la casa entre lo público y lo privado. Las secciones [Figura 6] permiten identificar el traslado de objetos a través de este límite, el cual se produce principalmente por tres motivos: la introducción de objetos de propiedad pública y uso colectivo al interior de la vivienda, el traslado temporal de objetos de propiedad privada hacia la calle, o la incorporación de mobiliario urbano municipal. Este libre desplazamiento de objetos a través de los límites conlleva, a su vez, a la apertura de los límites sociales impuestos por la familia o por el barrio, dando lugar a nuevas agrupaciones, asociaciones y escalas de colectividad.

Las cuatro secciones de la Figura 6 permiten ver en detalle los diferentes objetos y mobiliario doméstico de las casas y su contexto inmediato. Cada gráfico incorpora franjas de colores en la parte inferior; el color representa un uso particular del espacio. Asimismo, la ubicación de

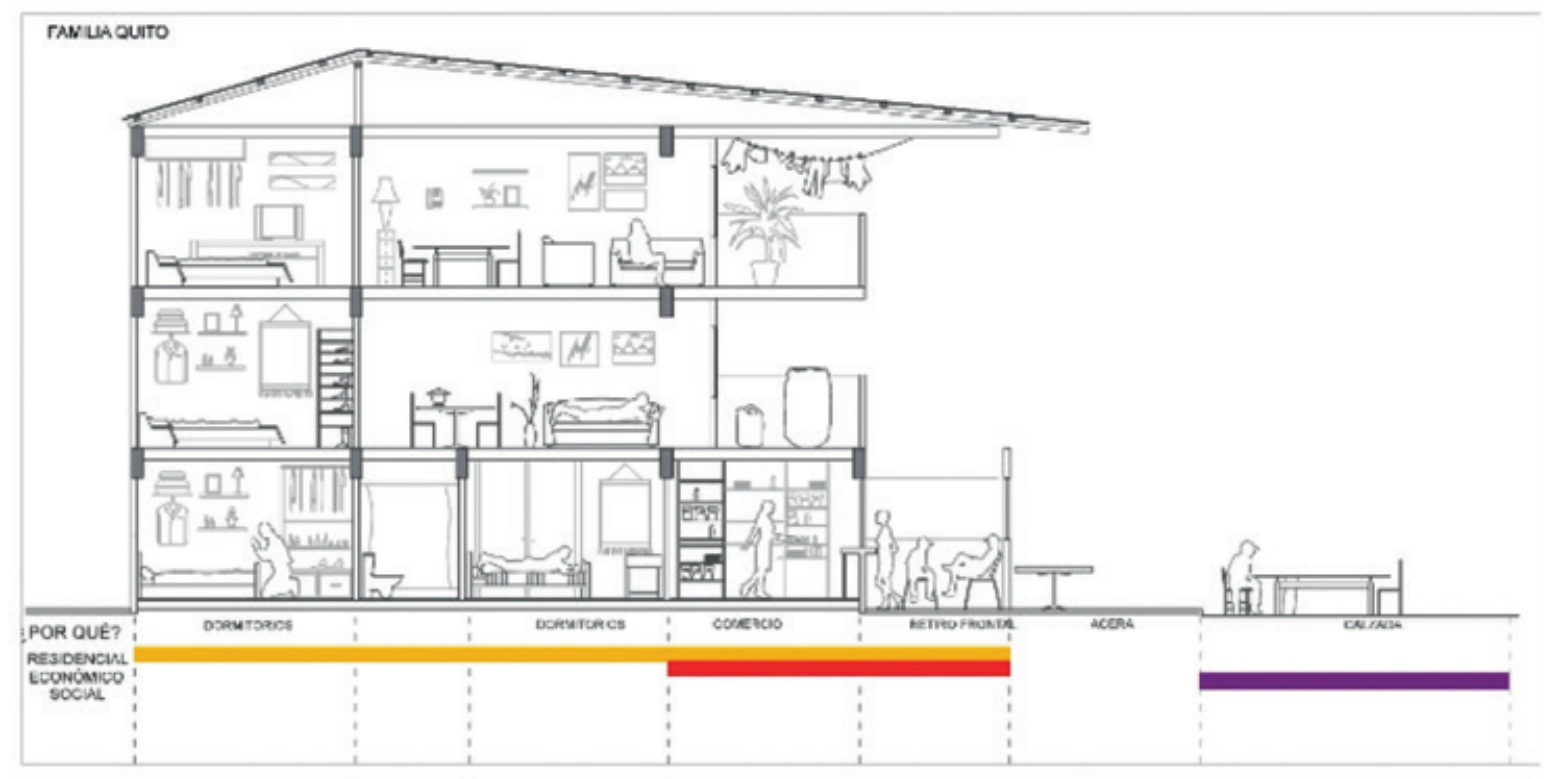


la franja permite visualizar — en proyección del dibujo superior- qué espacios de la sección son utilizados para actividades relacionadas con cada uso. Por ejemplo, el caso N² muestra cómo la franja amarilla, que corresponde al uso residencial, se extiende por toda la planta baja de la casa y se prolonga sobre la calle. En ese mismo caso, la franja roja, que ilustra el uso comercial, se extiende desde el soportal de la vivienda hacia el interior. Se produce por ello un traspaso del límite en ambas direcciones.

La lectura de las franjas permite observar la superposición de actividades de distinto uso en los mismos espacios, reforzando la disolución de distinciones entre el interior y el exterior, y entre lo público y lo privado. Además, revela la obsolescencia de un programa arquitectónico rígido, por cuanto no existe correspondencia entre actividades y espacios tradicionalmente definidos para un uso particular único. Este es el caso de la sección del caso $N^{\circ} 4$, que evidencia una franja roja de actividad comercial que ocupa más de la mitad del interior de la vivienda.
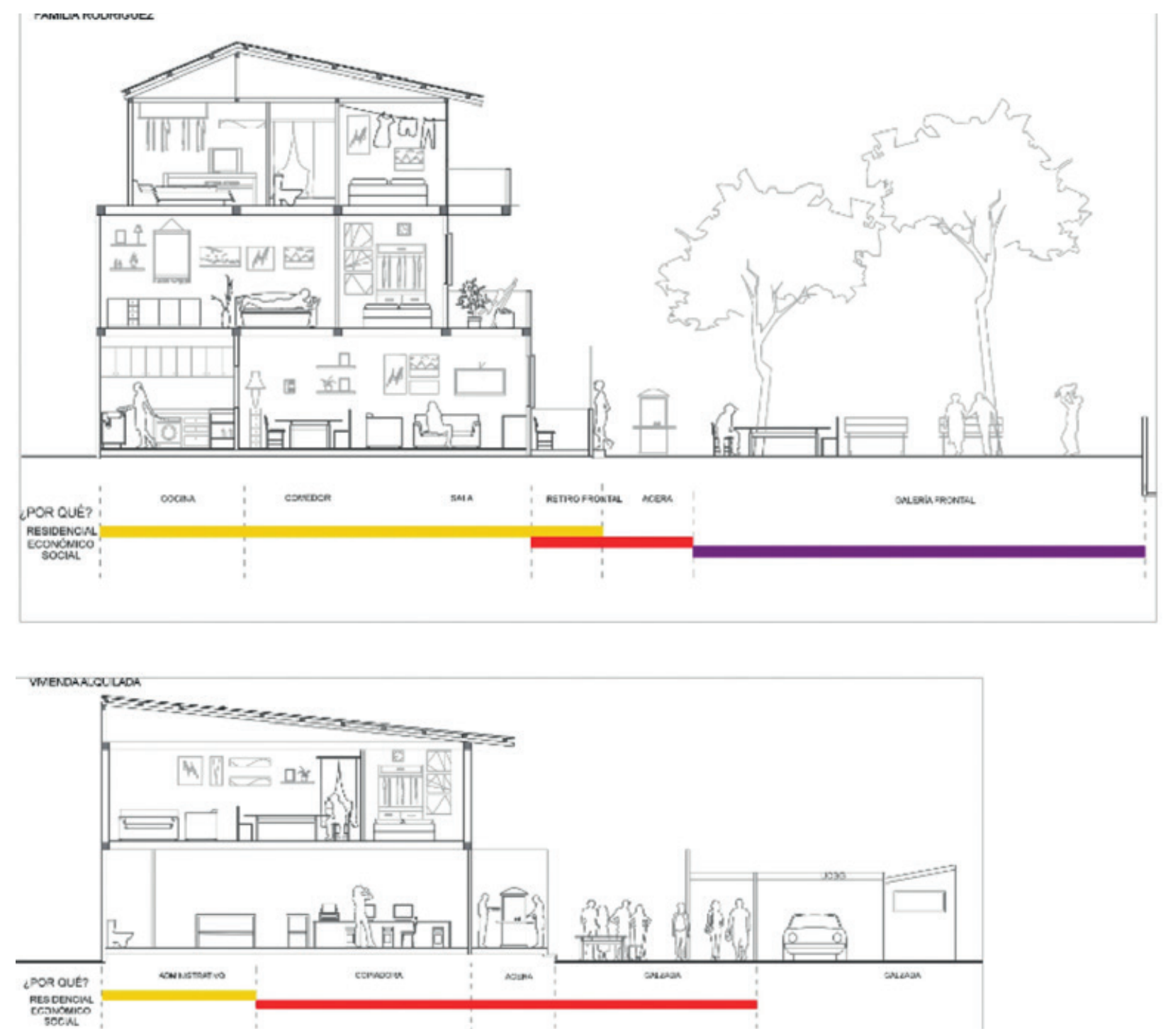


\section{PERSONAS, OBJETOS Y ACCIONES}

9 Yona Friedman, en sus "Structures serving the unpredictable" (1999), y Constant Nieuwenhuys, en su "Nueva Babilonia" (2009), describen este espacio formado por personas, objetos y acciones.

\section{DISCUSIÓN Y CONCLUSIONES}

\section{PRINCIPIO 1: \\ LA CASA COMO PUNTO DE ENCUENTRO}

10 Stan Allen (1999) habla de las "condiciones de campo" dentro de un sistema de elementos que se desplazan libremente.

11 El entendimiento de la casa como un refugio proviene sobre todo de la creación de una retórica de la seguridad y el miedo (Turner, 2017).
En la Figura 7 se han mapeado los desplazamientos de los integrantes de las cuatro familias durante la mañana, tarde y noche. Cada desplazamiento viene motivado por una acción que se asocia al uso de determinados objetos de la vivienda. Los colores representan las principales actividades que tienen lugar y los objetos involucrados en ellas. La superposición de los desplazamientos señala los puntos de encuentro entre los diferentes usuarios, delimitando los lugares de mayor afluencia.

La figura inferior derecha, en cada caso, señala estos centros de aglomeración, en donde se reúnen en algún momento del día un gran número de personas y objetos. En el caso $\mathrm{N}^{\circ} \mathrm{I}$, por ejemplo, la zona donde se sitúa el altar para la celebración de misas y los asientos para los asistentes queda resaltada como centro de mayor encuentro. Lo mismo ocurre en la zona de soportal del caso $\mathrm{N}^{\circ} 2$, o los locales comerciales del caso $\mathrm{N}^{\circ} 3$.

Esto nos lleva a entender la vivienda, no como una sumatoria de estancias funcionalmente diferenciadas, sino como un conjunto de objetos y personas que se reagrupan temporalmente. Los objetos y las personas forman, de esta manera, agrupaciones capaces de incorporar, perder o desplazar elementos. Tales acciones son las mismas que se producen en la estructura social de la familia, por lo que se posibilita una evolución paralela de ambos conjuntos.

Los resultados expuestos describen coincidentemente las necesidades que tuvieron las viviendas de todo el planeta durante los meses de confinamiento que demandó la pandemia: flexibilidad, adaptabilidad, participación, inmediatez, producción, etc. El objetivo de la investigación, que aquí se expone en parte, es precisamente describir esta lógica informal de transformación, que desde la precariedad en la que se desenvuelven las viviendas analizadas, permite extraer conclusiones aplicables a cualquier otro proyecto de vivienda.

Con ese propósito, se establecen a continuación lazos de unión entre estos resultados y varias posturas teóricas que se aproximan al territorio de los sistemas de objetos. Esto ha permitido definir seis principios fundamentales, susceptibles de ser exportados a nuevos casos.

La casa no es entendida como un volumen único que alberga un espacio habitable, sino como un "centro de masas" que conlleva una determinada densidad; un aglomerado de objetos que formaban parte anteriormente de otros conjuntos y que han sido recolocados y congregados alrededor de un centro de gravedad que los aglutina ${ }^{10}$ (Allen, 1999). De esta forma, la casa no viene definida tanto por un contorno físico (muros), ni legal (parcela), como por un "punto de encuentro" entre objetos y personas, que sustituye a sus límites ${ }^{11}$. La lectura de la casa como un conjunto de objetos se apoya fundamen- 


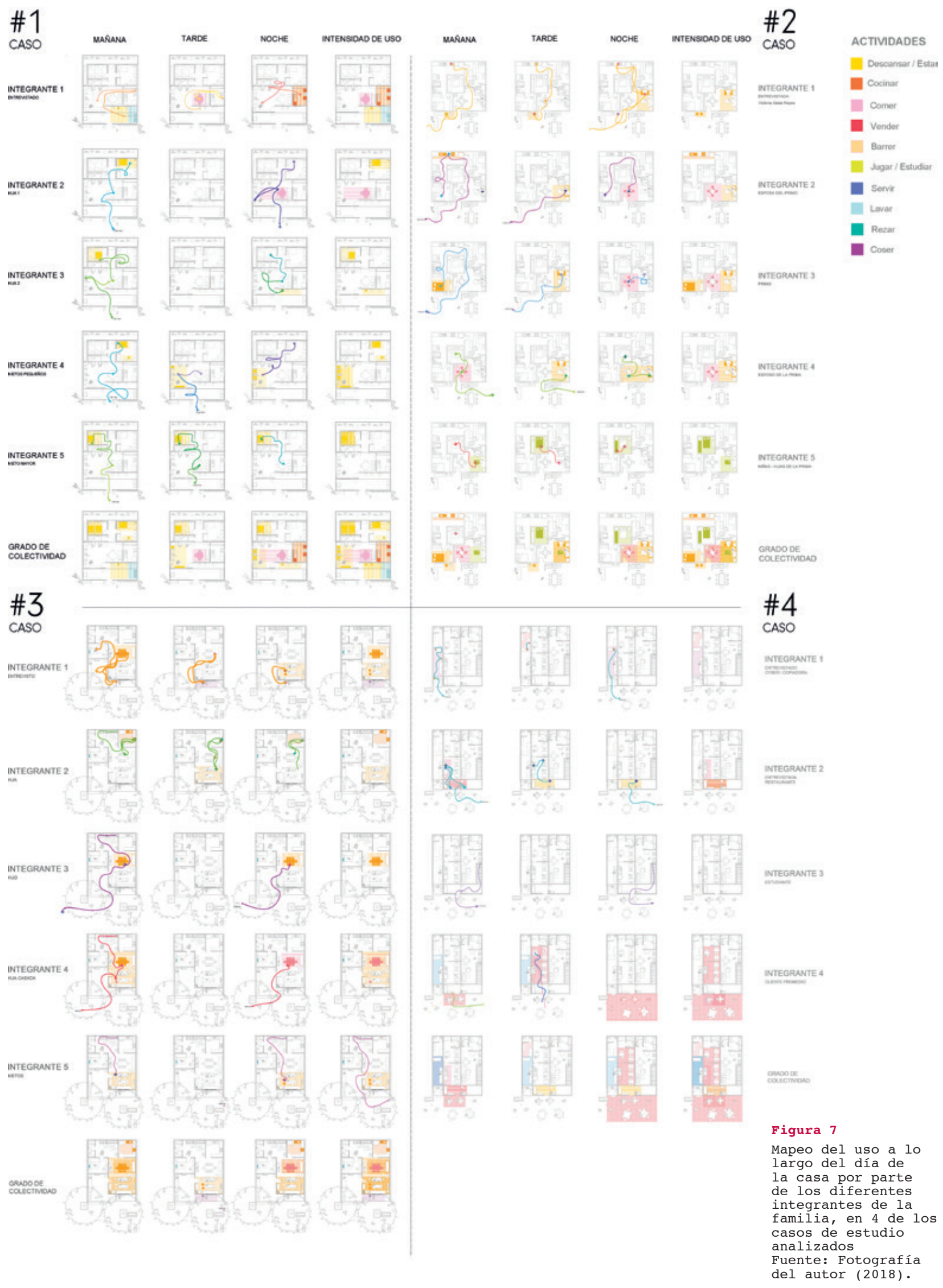




\section{PRINCIPIO 2: APROXIMACIONES FAMILIA-CASA}

\section{PRINCIPIO 3: \\ TRANSFORMACIÓN SOCIAL DE LA CASA}

12 Así lo describe la "planeidad" de Scott Lash (1999) y se opone al desequilibrio mostrado por el modelo "depredador-presa" de Wilensky y Resnick (1999). talmente en la "Teoría de los objetos" de Moles (1975), que arranca de la consideración de que los objetos cotidianos son capaces de formar un sistema de relaciones entre sí, como defiende Baudrillard en su "Sistema de los objetos" (1969). Se llega, inclusive, a entender que hay un cierta "vida social en las cosas" (Appadurai, 1991). En 1993, Latour le da nombre a estos sistemas de objetos y de personas a través de sus cuasi-objetos y cuasi-sujetos, pero es Lash (1999) quien equipara el sistema de los objetos al de las personas, demandando una necesaria "planeidad" entre ambos conjuntos.

La casa, al igual que la familia, resulta ser un acontecimiento expuesto también a contingencias a lo largo del tiempo (García-Huidobro, Tugas y Torres, 2008). Puede ser entendida, por tanto, como una "sociedad de objetos", con una naturaleza similar a la estructura social de la familia (Chombart de Lawe, 1960). Los objetos en esta sociedad se comportan sobre el suelo como personas, acercándose o alejándose, complementándose; son desplazados, incluidos en un grupo o en otro, etc.

Entender la vivienda como un sistema social de objetos posibilita, a su vez, la aparición de mecanismos de aproximación entre el sistema social de la familia y el sistema físico-social de la casa, dando lugar a un motor de cambio común (De Teresa, 20 I 6b). La familia y la casa tienen, según lo anterior, una estructura social parecida, por lo que pueden influenciarse mutuamente. Son entidades en permanente cambio, constituidas por sistemas de elementos (personas y objetos) que se transforman conjuntamente y que, en consecuencia, no pueden ser analizados por separado. Este sistema dual entre personas y cosas es, para algunos autores, más estable cuanto mayor igualdad de influencia haya entre ambos subsistemas ${ }^{12}$.

Los objetos de la casa pueden ser entendidos, desde esta perspectiva, como un "sistema social", con una estructura similar a la de la familia, por lo que pueden llegar a transformarse en paralelo. La casa tiene que ser capaz de transformarse siguiendo la misma lógica que la familia. No se trata sólo de crecer — como proponen los planes de vivienda "progresiva" - , sino de avanzar hacia un sistema global de vivienda cuya naturaleza sea capaz de afrontar la complejidad familiar y adaptarse a circunstancias diversas, entre ellas, las restricciones económicas, las relaciones cambiantes con el barrio y otras viviendas, y las de confinamiento obligatorio ejemplificadas, en la actualidad, con la pandemia del COVID-19.

La casa informal consolidada no pretende ser, por ende, un objeto puro y resistente a la transformación, sino un híbrido, que se comporta como uno de los "objetos indóciles" y difíciles de controlar que describe Lash (1999). En este sentido, el posible reciclaje no se debe producir a través del cambio físico de un objeto, sino del cambio en su posición y papel dentro de un conjunto. Lo mismo 


\section{PRINCIPIO 4: OBJETOS SUCIOS E HÍBRIDOS}

\section{PRINCIPIO 5: EL OBJETO COMO MEDIADOR}

13 Este artículo no propone excluir al arquitecto de la producción de vivienda, sino por el contrario, hacerle partícipe del $95 \%$ de las construcciones que actualmente prescinden de su labor.

14 Aproximadamente el $95 \%$ de las construcciones que se ejecutan en la ciudad de Guayaquil se desarrollan siguiendo un régimen informal, sin contar con la figura del arquitecto al suponerse un gasto prescindible (De Teresa, 2016b). ocurre con las familias, ya que no se transforman las personas, sino su manera de agruparse y el papel que desempeñan en un determinado grupo social. Son estos conjuntos, de personas o de objetos, los que se hibridan al desplazar e intercambiar elementos de unos grupos a otros.

Latour (1993) anuncia, en este contexto, una proliferación descontrolada de "monstruos híbridos" como consecuencia de la obra de purificación llevada a cabo por la modernidad. El autor describe como "objetos sucios" a todos aquellos que no pueden ser clasificados porque se encuentran en continua transformación. Esto sucede con las agrupaciones tanto de objetos, como de personas. Introduce así el concepto de "cuasi-sujetos" y "cuasi-objetos". Se trata de entidades formadas por elementos (personas o cosas) enlazados entre sí mediante un sistema de relaciones, que está en permanente estado de actualización. Las personas, como sujeto colectivo, y las cosas, como objeto colectivo, no se corresponden con tipologías claras: son híbridos que no encajan en ninguna taxonomía.

Los casos de estudio analizados ejemplifican esta variabilidad en la composición familiar, pues las estructuras familiares difieren enormemente unas de otras en cuanto a número de integrantes, papel que desempeñan dentro del conjunto (inquilinos, familiares lejanos, etc.), y núcleos familiares en los que se estructuran. Tanto la casa como la familia, tienden a convertirse por ello en los objetos y sujetos "sucios", híbridos e inclasificables, que describe el citado Latour, o en el difícil conjunto esbozado por Venturi (1978).

Los objetos son, como se ha visto, los principales responsables de las transformaciones de las viviendas. Es gracias al traslado de ciertos objetos que se generan actividades y encuentros entre las personas. Son ellos los primeros en apropiarse de un lugar, tanto dentro como fuera de la casa. Son también los encargados de cambiar el uso de un espacio o de hibridar usos al mezclarse entre sí. El objeto se convierte entonces, inevitablemente, en un mediador entre las personas y su entorno.

Es probablemente a través de estos objetos, la manera en que el arquitecto puede llegar a intervenir en estos contextos de precariedad $^{13}$. Los usuarios de las viviendas analizadas compran todo lo que hay en su casa, salvo una cosa: la propia casa. La arquitectura profesional que conocemos no está hecha para ser vendida en estos $\operatorname{casos}^{14}$. El papel principal del arquitecto, como profesional encargado de proyectar una vivienda, deba quizás incorporar este rol de "diseñador de producto", encargándose de idear objetos que puedan ser adquiridos por las familias e incorporados al elenco doméstico. La arquitectura tiene que poder ofrecer un producto asequible, y a través de él, aportar calidad a las viviendas. De este modo, el objeto se convierte en mediador entre el arquitecto y el usuario. 


\section{PRINCIPIO 6: ARQUITECTURA POR CATÁLOGO}

15 Muchos gremios han reaccionado frente a la pandemia, ofreciendo nuevos artículos de venta directa como mascarillas, purificadores de aire, mecanismos para abrir puertas sin tocarlas, etc. Estos objetos son ejemplo de cómo puede una industria determinada intervenir en cualquier hogar de manera inmediata.

16 Walter Benjamin analiza en "La obra de arte en la era de su reproducción mecánica" (2013), la capacidad del objeto industrializado de volverse verdaderamente trascendente.

17 En el terreno de la arquitectura de interiores, o de la moda, por ejemplo, la personalización no está ya en la contratación de un "decorador", o de un "modista", sino en la selección y combinación de piezas producidas por grandes almacenes como IKEA o ZARA, que democratizan sus diseños, haciéndolos accesibles. Ambos gremios (decoración y moda) han abandonado ya el campo del diseño personalizado, para llegar a las masas.

18 Tienen relación, desde esta lógica, con los "objetos democráticos" de Kenji Ekuan (Koolhaas, 2011), los "objetos que juzgan" de Latour y Lash o los "sistemas de juguete" de LEGO. Recae, de esta forma, sobre los objetos la responsabilidad de legislar e incluso de educar
Esta necesidad de cambio en el rol del arquitecto, no es sin embargo exclusiva de la vivienda informal. Tras la pandemia, el planeta entero demanda lo mismo que las viviendas informales analizadas: poder transformarse fácilmente frente a cualquier contingencia, haciendo partícipes directos a los propios usuarios. La única manera que tiene el arquitecto de intervenir en estas situaciones, y a nivel global, es igualmente a través del diseño de objetos que puedan ser comprados directamente por las familias ${ }^{15}$. La crisis de la vivienda actual demanda, en efecto, una revisión del papel del arquitecto, para poder mediar de manera indirecta, pero inmediata, en las alteraciones que pueda requerir cualquier hogar.

Esto supone incorporar el diseño de objetos a la práctica formal de los arquitectos dedicados al tema de la vivienda, generando una "arquitectura por catálogo", capaz de responder a la inmediatez de la vida urbana. La investigación sugiere, en este punto, la aparición de un hábitat formado por objetos fabricados en serie, que puedan ser adquiridos a buen precio y que puedan a la vez ser desplazados, intercambiados, desechados, etc. ${ }^{16}$. De esta forma, el catálogo convierte a los objetos cotidianos, ordinarios y vulgares en la clave para entender el mundo, para hacer "política de lo cotidiano" y para transformar, a través de ellos, la sociedad. No se trata de hacer una clasificación, sino un surtido, un menú, una caja de herramientas. Esta arquitectura por catálogo tiene como objetivo pasar de la cultura anti-híbrida, a la aceptación de una proliferación de híbridos, que comienza con la propia proliferación de los catálogos. Esto permitiría una mayor diversidad y, por lo tanto, una mayor capacidad de elección y de personalización, al igual que sucede con la ropa de nuestro armario o con los muebles de nuestra habitación ${ }^{17}$.

En este mundo de objetos en serie, cada uno lleva implícitas ciertas posibilidades de transformación, las cuales funcionan como "normas sociales" propias, encargadas de regular el comportamiento del conjunto ${ }^{18}$. Son estos objetos el camino para la innovación en el campo de la vivienda, al ser capaces por sí solos de introducir cambios en cualquier hogar (panel solar, compostera, balcón...) o al hibridarse con otros objetos, trasladando al usuario la capacidad de innovar. El campo de acción de estos objetos puede entonces extenderse a lo largo de todas las escalas, desde los pequeños objetos de un cajón, hasta la escala de la casa, o incluso de la ciudad, convirtiéndolos en protagonistas del escenario doméstico y urbano. Los objetos son los responsables, en definitiva, de traspasar los límites físicos impuestos por la casa y los límites sociales impuestos por la familia, y de tejer una densa estructura social capaz de formar un motor de cambio conjunto. 


\section{REFERENCIAS BIBLIOGRÁFICAS}

ALLEN, S. (1999). From object to field. Recuperado de: http://lostritto. com/risd2013spring/wp-content/uploads/2013/04/allen1.pdfv.

APPADURAl, A. (1991). La vida social de las cosas. Perspectiva cultural de las mercancías. México: Grijalbo.

BAUDRILLARD, J. (1969). El sistema de los objetos. Madrid: Siglo XXI.

BENJAMIN, W. (2013). La obra de arte en la época de su reproducción mecánica. Madrid: Casimiro.

CHOMBART DE LAUWE, P. (1960) Famille et Habitation. París, CNRS.

DE MOLINA, S. (2013). La invasión de los objetos. Recuperado de: http:// www.santiagodemolina.com/2013/01/la-invasion-de-los-objetos.html.

DE MOLINA, S. (2014). Collage y Arquitectura: la forma intrusa en la construcción del proyecto moderno. Sevilla: Recolectores Urbanos.

DE TERESA, I. (2015). Relación entre las características tipológicas funcionales de la vivienda unifamiliar informal y su evolución espacial. Caso: Santa María de las Lomas, Guayaquil. Guayaquil: SINDE, UCSG.

DE TERESA, I. (2016a). Aproximaciones Familia-Casa: Ia Vivienda Informal Consolidada en Santa María de las Lomas, Guayaquil. Dearq Revista de Arquitectura, (19), 30-43.

DE TERESA, I. (2016b). Transformaciones incrementales en la vivienda informal consolidada: el caso de Santa María de las Lomas, Guayaquil. Arquitecturas del Sur, 34(49), 6-21.

FRIEDMAN, Y. (1999). Structures serving the unpredictable, Barcelona, Nai Publishers.

GARCÍA-HUIDOBRO, F., TUGAS, N. Y TORRES, D. (2008). El tiempo construye. Lima: Gustavo Gili.

HERNÁNDEZ, F., KELLET, P. Y ALLEN, L.K. (2012). Rethinking the Informal City. Critical perspectives from Latin America.Nueva York: Berghahn Books. IGLESIA, R. (2011). La vida doméstica y los objetos. Buenos Aires: IAA. Recuperado de: http://www.iaa.fadu.uba.ar/publicaciones/critica/0165.pdf KOOLHAAS, O. (2011). Project Japan. Metabolism Talks. Chicago: Taschen. LASH, S. (1999). Objetos que juzgan: el parlamento de las cosas de Latour. Recuperado de: http://eipcp.net/transversal/0107/lash/es.

LATOUR, B. (1993). We have never been modern. Cambridge: Harvard University Press.

MOLES, A. (1975). Teoría de los objetos. Barcelona: Gustavo Gili.

MORA, E., VITERI, F. Y DE TERESA, I. (2017). Estudio de la Generación de Colectividad en la Vivienda Informal Consolidada". Caso: Santa María de las Lomas, Guayaquil. Guayaquil: SINDE, UCSG.

MORA, E. (2013). Proceso de crecimiento progresivo de las viviendas y su relación con factores y características de cambio en la estructura y dinámica de las familias de menores ingresos. Guayaquil: SINDE, UCSG.

NIEUWENHUYS, C. (2009). La Nueva Babilonia. Barcelona: GG mínima.

ROSLING, H. (2019). Factfulness. Barcelona: Deusto.

TURNER, J. (2017). John Turner: por una autonomía del habitar. Escritos sobre vivienda, autogestión y holismo. Logroño: Pepita de Calabaza Editores.

VENTURI, R. (1978). Complejidad y contradicción en la arquitectura. Barcelona: Gustavo Gili.

WILENSKY, U. Y RESNICK, M. (1999). Thinking in Levels: a Dynamic Systems Approach to Making Sense of the World. Journal of Science Education and Technology, 8(1), 13-19. 